\title{
A Gas and Dust Rich Giant Elliptical Galaxy
}

\author{
O. Krause ${ }^{1}$, U. Lisenfeld ${ }^{2}$, U. Klaas ${ }^{1}$, D. Lemke ${ }^{1}$, M. Haas ${ }^{1}$, M. Stickel ${ }^{1}$ \\ ${ }^{1}$ Max-Planck-Institut für Astronomie, Königstuhl 17, 69117 Heidelberg, \\ Germany \\ ${ }^{2}$ Instituto de Astrofisica de Andalucia, Camino Bajo de Huetor 24, \\ 18080 Granada, Spain
}

\begin{abstract}
The bolometric luminosity of $L_{\mathrm{FIR}}=2 \times 10^{12} \mathrm{~L}_{\odot}$ makes ISOSS $\mathrm{J} 15079+7247$ one of the most luminous and unusual galaxies detected by the $170 \mu \mathrm{m}$ ISOPHOT Serendipity Survey (ISOSS). The detection of CO (1-0) emission identifies a giant elliptical galaxy at redshift $z=0.2136$ as the counterpart of the FIR source. The derived high gas mass of $3 \times 10^{10} \mathrm{M}_{\odot}$ favours the picture that the dust emission is associated with this elliptical galaxy. The ultraluminous IR emission can be explained by a hidden starburst in the center of the elliptical. This is supported by the strength of non-thermal radio continuum emission. The huge dust mass of $5 \times 10^{8} \mathrm{M}_{\odot}$ corresponds to a visual extinction of $A_{\mathrm{V}} \sim 1000 \mathrm{mag}$, being consistent with the non-detection of any signatures of a strong starburst in ISOSS J $15079+7247$ in optical spectra.
\end{abstract}

\section{Introduction}

The ISOPHOT Serendipity Survey (ISOSS) (Bogun et al. 1996) has observed about 2000 galaxies at $170 \mu \mathrm{m}$, providing a unique data base of far-infrared spectral energy distributions beyond the IRAS $100 \mu \mathrm{m}$ limit. Stickel et al. (2000) found that late type galaxies generally contain a cold $(T \sim 20 \mathrm{~K})$ dust component over a wide range of infrared luminosities $\left(10^{9}-10^{11} \mathrm{~L}_{\odot}\right)$. For early type galaxies, however, the traditional view is that they are less dusty and less luminous in the infrared (see Knapp 1999 for a review). We summarize here a detailed study of the elliptical galaxy ISOSS $J 15079+7247$, detected by the ISOPHOT Serendipity Survey as an ultraluminous infrared source (Krause et al. 2003).

\section{The optical counterpart of ISOSS J $15079+7247$}

The ISOSS source coincides in position with a compact $1.2 \mathrm{~mm}$ continuum source detected with the MAMBO bolometer array. The astrometric accuracy of our MAMBO observations allowed to identify an elliptical galaxy as the optical counterpart of the FIR/millimetre source (Fig. 1). Optical long slit spectroscopy revealed a pure absorption spectrum towards the nucleus. The redshift derived from the $\mathrm{Ca} \mathrm{H} \& \mathrm{~K}$, G-band and Fe $5340 \AA$ features is $z=0.2137 \pm 0.0003$. The detection of $\mathrm{CO}(1-0)$ emission at the optical redshift of the elliptical galaxy strongly suggests that the molecular gas is physically associated with this galaxy. The very large molecular hydrogen mass of $M\left(\mathrm{H}_{2}\right)=2.9 \times 10^{10} \mathrm{M}_{\odot}$ is remarkable. 
So far, significantly smaller amounts of molecular gas have been observed in ellipticals (eg. Henkel \& Wiklind 1997).

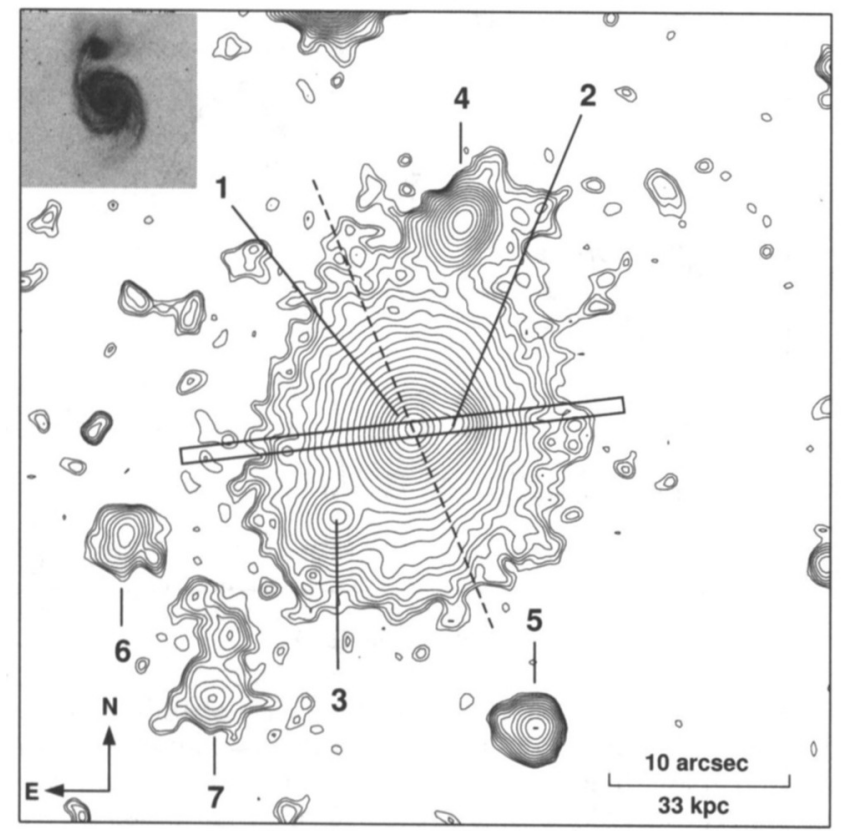

Figure 1. R-Band image of ISOSS J $15079+7247$ obtained with the LAICAcamera at the Calar Alto $3.5 \mathrm{~m}$ telescope. The giant elliptical galaxy (1) has a close companion (2) and shows a faint tail of emission (3) towards southeast. The slit position of the spectroscopic observations is indicated (box).

\section{Evidence for a starburst in the elliptical galaxy}

The far-infrared to millimetre spectral energy distribution (Fig. 2) can be well fitted by an optically thick modified blackbody of $42 \mathrm{~K}$, optical depth $\tau_{100 \mu \mathrm{m}}=$ 3.5 and dust emissivity $\beta=1.5$, yielding a dust mass of $M_{\mathrm{d}}=5.4 \times 10^{8} \mathrm{M}_{\odot}$. The source was barely resolved at the VLA in the cm-continuum (Condon et al. 1998) and the deconvolved size of $\sim 4.8 \times 1.6 \mathrm{kpc}^{2}$ may be considered as an upper limit for the starburst size, in agreement with our lower limit $r_{\mathrm{b}}=0.6 \mathrm{kpc}$ from the far-infrared. Assuming an average radius of $1 \mathrm{kpc}$ for the nuclear starburst and spherical distribution of dust and gas, we find a total column density of $N(\mathrm{H})=$ $1.7 \times 10^{24} \mathrm{~cm}^{-2}$ towards the center. This corresponds to a visual extinction of $A_{\mathrm{V}} \sim 1000 \mathrm{mag}$ and explains the absence of any optical emission lines towards the center of the elliptical, which is very unusal for ULIRGs (Veilleux et al. 1999). ULIRGs normally show a much more disturbed morphology and emission line spectra in their centers. ISOSS J $15080+7248$ may be an elliptical galaxy merging with a gas rich spiral inducing a powerful starburst in the center of the elliptical. The large amount of molecular gas and dust detected in the elliptical 
galaxy ISOSS J $15079+7247$ suggests an alternative explanation of the nature of several other high-redshifted submm-galaxies.

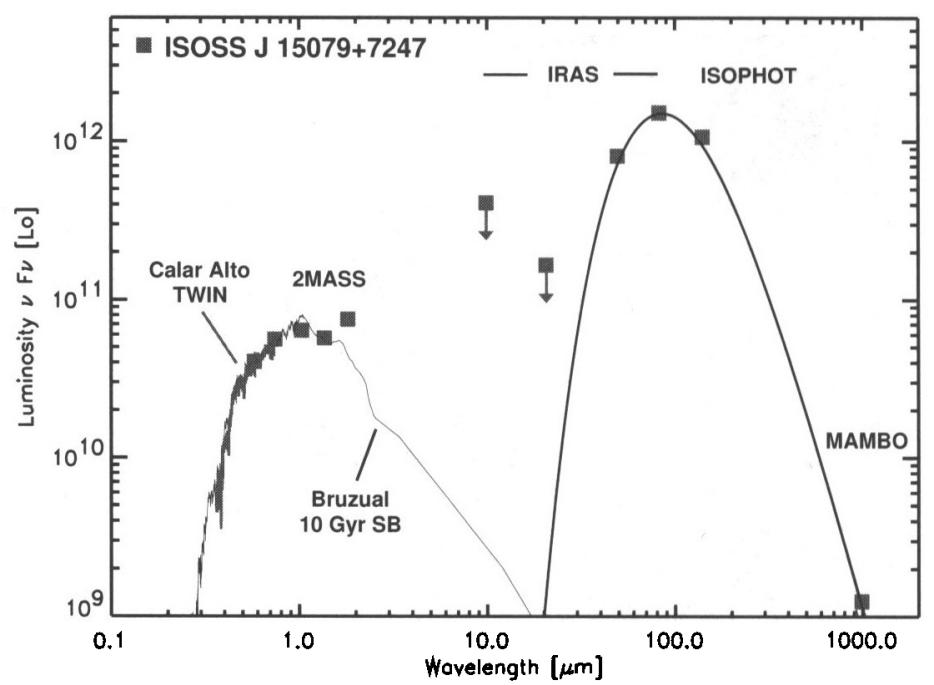

Figure 2. Rest frame spectral energy distribution of ISOSS J $15079+7247$. The FIR dust emission can be well characterized by an optically thick modified blackbody (solid line). The optical and near-infrared emission can be fitted by a 10 Gyr old starburst following Bruzual \& Charlot 1995.

Acknowledgments. The ISOPHOT Data Center at MPIA is supported by Deutsches Zentrum für Luft- und Raumfahrt e.V. (DLR) with funds of Bundesministerium für Bildung und Forschung, grant no. 50QI0201. OK thanks the Wernher von Braun-Stiftung zur Förderung der Weltraumwissenschaften e.V. for financial support.

\section{References}

Bogun, S., Lemke, D., Klaas, U. et al. 1996, A\&A, 315, L71

Bruzual, G.A. \& Charlot, S. 1995, Starburst Template Library in IRAF-STSDAS

Condon, J.J., Yin, Q.F., Thuan, T.X. et al. 1998, ApJ, 116, 2682

Henkel, C. \& Wiklind, T. 1997, Space Science Reviews, 81, 1

Knapp, G.R. 1999, in ASP Conf. Ser. 163, Star Formation in Early-Type Galaxies, ed. P. Carral \& J. Cepa, (San Francisco, ASP), 119

Krause, O., Lisenfeld, U., Lemke, D. et al. 2003, A\&A, 402, L1

Stickel, M., Lemke, D., Klaas, U. et al. 2000, A\&A, 359, 865

Veilleux, S., Kim, D.-C. \& Sanders, D.B. 1999, ApJ, 522, 113 